Hamid Motallebzadeh, Mathieu Charlebois, and W. Robert J. Funnell

\title{
A non-linear viscoelastic model for the tympanic membrane
}

Published in:

Journal of the Acoustical Society of America, 134(6), December 2013.

Pp. 4427-4434

Copyright (2013) Acoustical Society of America. This article may be downloaded for personal use only. Any other use requires prior

permission of the author and the Acoustical Society of America.

doi: $10.1121 / 1.4828831$

http://scitation.aip.org/content/asa/journal/jasa/134/6/10.1121/1.4828831

Uploaded to eScholarship@mcgill.ca on 8 June 2015. All items in eScholarship@McGill are protected by copyright with all rights reserved unless otherwise indicated. 


\title{
A non-linear viscoelastic model for the tympanic membrane
}

\author{
Hamid Motallebzadeh and Mathieu Charlebois \\ Department of BioMedical Engineering, Faculty of Medicine, McGill University, 3775 rue University, \\ Montréal, Québec H3A 2B4, Canada \\ W. Robert J. Funnell \\ Departments of BioMedical Engineering and Otolaryngology-Head \& Neck Surgery, McGill University, \\ 3775 rue University, Montréal, Québec H3A 2B4, Canada
}

(Received 14 April 2013; revised 12 September 2013; accepted 21 October 2013)

\begin{abstract}
The mechanical behavior of the tympanic membrane displays both non-linearity and viscoelasticity. Previous finite-element models of the tympanic membrane, however, have been either non-linear or viscoelastic but not both. In this study, these two features are combined in a non-linear viscoelastic model. The constitutive equation of this model is a convolution integral composed of a non-linear elastic part, represented by an Ogden hyperelastic model, and an exponential time-dependent part, represented by a Prony series. The model output is compared with the relaxation curves and hysteresis loops observed in previous measurements performed on strips of tympanic membrane. In addition, a frequency-domain analysis is performed based on the obtained material parameters, and the effect of strain rate is explored. The model presented here is suitable for modeling large deformations of the tympanic membrane for frequencies less than approximately $3 \mathrm{rad} / \mathrm{s}$ or about $0.6 \mathrm{~Hz}$. These conditions correspond to the pressurization involved in tympanometry.

(C) 2013 Acoustical Society of America. [http://dx.doi.org/10.1121/1.4828831]
\end{abstract}

PACS number(s): 43.64.Ha, 43.64.Bt [KG]

Pages: $4427-4434$

\section{INTRODUCTION}

The eardrum or tympanic membrane (TM), the gateway to the middle ear, has a curved conical shape with the apex pointing medially. It receives airborne sound waves collected by the outer ear, transforms them into mechanical vibrations, and transmits the vibrations to the ossicular chain. Changes of the structure and properties of the TM directly affect the sound transmission and may lead to conductive hearing loss. In addition, the response of the TM greatly influences the results of hearing screening and diagnosis tests. For example, in tympanometry the TM contributes more to the overall response than other middle-ear components do (e.g., Feldman, 1974). Thus, understanding the mechanics of the TM is essential for understanding conductive hearing loss and for designing better screening and diagnostic tests. It is also important for TM repair procedures and for improvement of the coupling between ossicular prostheses and the TM.

The small dimensions and non-uniform thickness of the TM make measurements of its mechanical properties very challenging. Added to these difficulties are the non-linear stress-strain relationship and strain-rate-dependent behavior of the TM.

In the literature, various measurements of the mechanical properties of the TM have been reported, in association with various models. Békésy (1949) measured the elastic modulus (or Young's modulus) of the human TM using a bending test on a rectangular flap. Kirikae (1960) calculated the elastic modulus based on a dynamic test on a strip of fresh human TM. Decraemer et al. (1980a, 1980b) reported data on the viscoelasticity of the TM and presented nonlinear elastic and non-linear viscoelastic structural models.
Fay et al. (2005) applied several approaches to estimate the elastic properties of the TM. Cheng et al. (2007) conducted uniaxial tensile tests on strips of fresh human TMs to measure the stress-stretch relationship, stress relaxation under constant deformation, and mechanical strength. They also applied a non-linear elastic model to the experimental data to analyze the non-linear stress-stretch behavior.

More recently, Huang et al. (2008) and Daphalapurkar et al. (2009) used a nanoindentation method to measure both in-plane and through-thickness viscoelastic properties of the posterior and anterior portions of the human TM. The applied deformations and resultant strains were small and linear. Luo et al. (2009a, 2009b) used a split-Hopkinson pressure bar to measure the strain-rate-dependent behavior of the normal and diseased human TM at strain rates of $300-2000 \mathrm{~s}^{-1}$ in the radial and circumferential directions. At these strain rates the reported stress-strain curves are linear except in the vicinity of failure strains. Zhang and Gan (2010) stimulated human TM specimens by sound pressure and measured the vibrations with a laser Doppler vibrometer. The sound pressure was $80 \mathrm{~dB}$ sound pressure level, which is within the range of linear TM vibrations (e.g., Khanna and Tonndorf, 1972). Most recently, Aernouts and Dirckx (2012) performed in situ sinusoidal indentations on gerbil TMs at frequencies from 0.2 to $8.2 \mathrm{~Hz}$. The strain magnitudes were small enough that the responses were linear. In all of these recent studies, therefore, the behavior of the TM was effectively linear and linear viscoelastic models are applicable.

For large deformations, however, the TM displays both non-linear and viscoelastic behavior. Previous finite-element models of the TM, however, have included either non-linear behavior (e.g., Ladak et al., 2006; Qi et al., 2008) or viscoelastic behavior (e.g., Zhang and Gan, 2010) but not both. In 
an earlier study (Charlebois et al., 2013) we investigated two approaches to modeling nonlinear viscoelastic behavior numerically. In this study, we use one of those approaches to combine non-linear elasticity (hyperelasticity) and viscoelasticity of the TM in a non-linear viscoelastic (NLV) finiteelement model. We validate our model against the viscoelastic relaxation data and the non-linear stretch data reported by Cheng et al. (2007). Our approach allows us to model both the loading and unloading curves, and the associated hysteresis, with a single set of parameters. We also estimate the energy dissipation as a function of frequency, and explore the effects of strain rate on the relaxation behavior.

\section{METHODS}

\section{A. Experimental data}

Details of the mechanical test procedures can be found in the paper of Cheng et al. (2007). Rectangular strips were cut from the posterior regions of human TMs, almost parallel to the manubrium, using a knife with two parallel blades $2 \mathrm{~mm}$ apart. The rectangular strips were flattened and mounted in a material-testing system and uniaxial tests were done.

The measurements were performed under controlleddeformation conditions. However, there is a discrepancy between their use of the term "strain rate" and their specification of $\mathrm{mms}^{-1}$ as the units. Strain rate is defined as $\dot{\varepsilon}_{i, j}$ $=\mathrm{d} \varepsilon_{i, j} / \mathrm{d} t$ (in units of $\mathrm{s}^{-1}$ ) while in a controlled-deformation experiment an elongation rate $i=\mathrm{d} l / \mathrm{d} t$ (in units of $\mathrm{mm} \mathrm{s}^{-1}$ ) is the input for the test. We have confirmed with them that the values given in their paper as 0.1 and $1.8 \mathrm{~mm} \mathrm{~s}^{-1}$ were actually elongation rates and not strain rates. Strain rate is calculated by dividing the elongation rate by the initial length (i.e., $\dot{\varepsilon}_{i, j}=\mathrm{d} \dot{l} / l_{0}$ ).

The lengths, widths, and thicknesses of the 11 TM specimens were 5.0-8.5 mm (mean 6.44), 1.5-2.4 mm (mean 1.97 ), and $0.06-0.1 \mathrm{~mm}$ (mean $0.08 \mathrm{~mm}$ ), respectively. Excluding a failure test that is not considered here, two testing protocols were performed by Cheng et al. (2007). First, in the standard uniaxial tensile test (stress-stretch relationship measured with displacement-ramp loading and unloading), the elongation rate was set at $0.1 \mathrm{~mm} \mathrm{~s}^{-1}$ and the maximum elongation was $15 \%$ of the original length. Second, in the stress relaxation test (stress measured as a function of time with rapid displacement-ramp loading followed by maintenance of the displacement), the elongation rate was set at $1.8 \mathrm{~mm} \mathrm{~s}^{-1}$ and the maximum elongation was again $15 \%$ of the original length (i.e., stretch $=1.15$ ). Loading time and final length are calculated here based on average dimensions because the individual dimensions were not given by Cheng et al. (2007).

\section{B. Finite-element model}

The TM is a complex structure composed of multiple layers (e.g., Lim, 1995). The orientation of collagen fibers in radial and circumferential directions in separate layers, in addition to a nonuniform thickness (e.g., Kuypers et al., 2006), make it inhomogeneous and anisotropic. However, no details are available concerning the thickness and other variations in the TM strips of Cheng et al. (2007). In this study, therefore, as in many other studies, the TM strips are assumed to be homogeneous and isotropic with constant thickness.

In addition, the TM is approximately conical so the rectangular strips of experimental specimens are not flat. For a flexible and thin membrane (thickness/width $<0.05$ and thickness/length $<0.02$, for the average dimensions of the TM strips) the bending moments are of minor importance (Schomburg, 2011). Therefore, as in Cheng et al. (2007), we can neglect the effects of flattening the TM strips.

A three-dimensional finite-element model of a rectangular TM specimen has been created in order to simulate the experimental data. The length, width, and thickness of the TM strip have been taken to have the mean values mentioned above $(6.44,1.97$, and $0.08 \mathrm{~mm}$, respectively). Taking advantage of symmetry to reduce computational cost, just one eighth of the actual TM strip has been modeled. The model consists of $800(20 \times 10 \times 4)$ isoparametric eightnode hexahedral (brick) elements with linear shape functions (Fig. 1). Since the elements have a poor aspect ratio, a much finer mesh, with elements of the same type but having a 1:1:1 (cubic) aspect ratio $(644 \times 196 \times 8$ elements $)$, was used to evaluate the adequacy of the coarse mesh. The simulation results with the coarse mesh and with the finer mesh

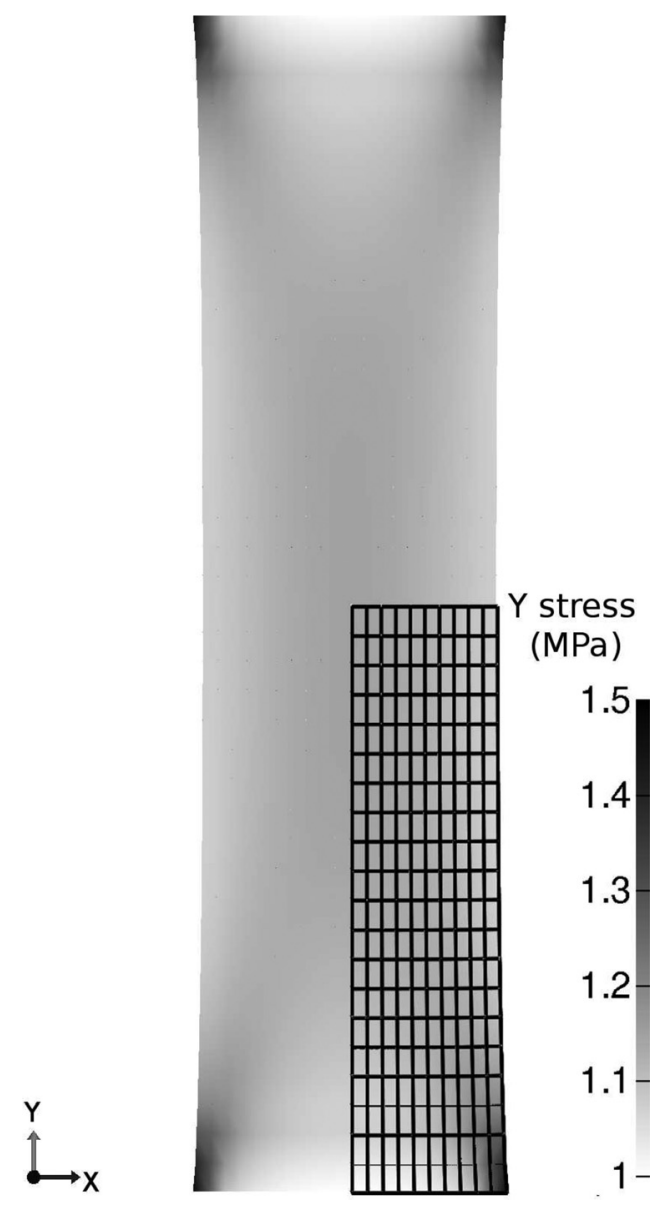

FIG. 1. Meshed one-eighth model of a TM strip. Taking advantage of the symmetry of the strip, the overall stress distribution is determined based on that of the one-eighth model. 
were found to differ by less than $2.4 \%$. The experiments are simulated by clamping one end of the model and displacing the other end by specified amounts.

The finite-element simulations are performed using the open-source software FEBio (Maas et al., 2012) (version 1.5.1) and it pre-processor PreView (version 1.4) and postprocessor PostView (version 1.3.5). FEBio is a nonlinear finite-element solver specifically designed for biomechanical applications. It uses an implicit time-integration scheme with an incremental iterative strategy based on Newton's method for nonlinear energy functions.

\section{Constitutive equations}

Different approaches have been used in the literature to derive the constitutive equations for non-linear viscoelastic materials. In this study we assume linear viscosity and nonlinear elasticity (hyperelasticity). The material response is expressed as a convolution of a time-dependent component and an elastic component, similar to what is done for linear viscoelasticity. We refer here to this model as a NLV model. FEBio calculates the total second Piola-Kirchhof stress tensor $\boldsymbol{S}(t)$ by convolving a normalized relaxation function $G(t)$ with the derivative of an elastic response function $S^{e}$ :

$$
\boldsymbol{S}(t)=\int_{0}^{t} G(t-u)\left(\frac{\mathrm{d} \boldsymbol{S}^{e}}{\mathrm{~d} u}\right) \mathrm{d} u
$$

where $t$ is time and $u$ is a dummy variable. The Prony series is a commonly used form for $G(t)$. This formulation not only facilitates interconversion of viscoelastic functions between the time and frequency domains but also increases the efficiency of discretization procedures in numerical methods (e.g., Taylor et al., 1970; Park and Schapery, 1999). Depending on whether $S^{e}$ represents the instantaneous or long-term elastic response, $G(t)$ is given by either

$$
G(t)=1-\sum_{i=1}^{N} g_{i}\left(1-\exp \left(-t / \tau_{i}\right)\right)
$$

or

$$
G(t)=1+\sum_{i=1}^{N} g_{i} \exp \left(-t / \tau_{i}\right)
$$

respectively. In both equations, $g_{i}$ (relaxation coefficients) and $\tau_{i}$ (time constants) are material parameters and $N$ is the number of exponential terms.

Among the many constitutive models that have been proposed for hyperelastic materials, the Ogden model is popular for biological tissues (e.g., Martins et al., 2006). According to this model, a strain energy $W$ composed of deviatoric and volumetric energies (i.e., energies due to distortion and to change of volume, respectively) is defined by

$$
W=\sum_{j=1}^{M} \frac{2 \mu_{j}}{\alpha_{j}^{2}}\left[\bar{\lambda}_{1}^{\alpha_{j}}+\bar{\lambda}_{2}^{\alpha_{j}}+\bar{\lambda}_{3}^{\alpha_{j}}-3\right]+W_{\mathrm{vol}}(J),
$$

where $J=\lambda_{1} \lambda_{2} \lambda_{3}$ is the determinant of the elastic deformation gradient; $M$ is the number of Ogden terms used; $\bar{\lambda}_{i}=\lambda_{i} / J^{1 / 3}$ are modified stretches, where $\lambda_{i}$ are the stretches; $\alpha_{j}$ and $\mu_{j}$ are material coefficients (with $\mu_{j}>0$ for thermodynamic consistency); and $W_{\mathrm{vol}}(J)$ is the volumetric part of the energy function. [This formulation is slightly different from that of Ogden (1972), where $\alpha_{j}$ was used instead of $\alpha_{j}^{2}$. The difference between these two formulations was discussed by Charlebois et al. (2013).] In this study we use only a single Ogden term (i.e., $M=1$ ).

Material parameters for a hyperelastic material can be determined by performing an unconfined tensile or compression test (to determine the deviatoric properties) and a confined compression test (to determine the volumetric part of the energy function). In the study from which we are taking our experimental data (Cheng et al., 2007), the mechanical tests were performed uniaxially on strips cut from TMs. Therefore, determining a volumetric response is not possible. Since soft tissue is generally assumed to be nearly incompressible (e.g., Humphrey, 2003), we model the TM as an incompressible material by setting the bulk modulus high enough to make $W_{\text {vol }}$ negligible. For a uniaxial tensile test, the resultant stress is related to the stretch by

$$
S_{1}^{e}=\frac{1}{\lambda_{1}} \frac{\partial W}{\partial \lambda_{1}}
$$

where the variables are the same as in Eqs. (1) and (4), and the subscript " 1 " indicates the loading direction. Note that, although in Eq. (1) the second Piola-Kirchhof stress is used for the elastic response function (i.e., $S^{e}$ ), the simulation output is given by FEBio as Cauchy (or "true") stresses. Furthermore, the experimental data of Cheng et al. (2007) were reported as nominal stresses. Thus we convert all stress measures to Cauchy stresses, $\sigma_{1}$, for the purposes of our analysis. The second Piola-Kirchhof stress and the nominal stress can be converted to Cauchy stress for the case of uniaxial loading by

$$
\sigma_{1}=\lambda_{1}^{2} S_{1} \quad \text { and } \quad \sigma_{1}=\lambda_{1} P_{1}
$$

respectively, where $P_{1}$ is the nominal stress.

\section{Determination of model parameters}

\section{Time-dependent part}

A common practice is to take one time constant per decade of data along the logarithmic time axis (e.g., Knauss and Zhao, 2007). This corresponds to one time constant per decade in the frequency domain as well (e.g., Puso and Weiss, 1998; Charlebois et al., 2013). To estimate the parameters, we first digitized the mean normalized relaxation curve reported for nine TM specimens (Cheng et al., 2007, Fig. 8) at the fifteen points where mean values and standard deviation bars were reported. Based on the intervals between those data points (1-10 s) and on the relaxation duration (the stress relaxation reaches a relatively stable state in $120 \mathrm{~s}$ ), a Prony series with three time constants (i.e., $1 \leq \tau_{1}<10$, $10 \leq \tau_{2}<100$, and $100 \leq \tau_{3}$ ) seems to be appropriate.

The Trust-Region non-linear least-squares method was used in the cftool curve-fitting function in MATLAB version 
7.8 (The MathWorks, Natick, MA) to identify material parameters. Each term in a Prony series involves the two parameters $g_{i}$ and $\tau_{i}$, resulting in two degrees of freedom (DOFs). For the three-term series, two approaches have been taken, one with six DOFs (three time constants and three relaxation coefficients) and one with three DOFs (the three relaxation coefficients) and three time constants $\tau_{i}$ that are predefined as 1, 10, and $100 \mathrm{~s}$. The use of predefined time constants reduces the computational cost and also reduces the need for defining constraints on the values of the parameters. To investigate whether three time constants are really required for the Prony series, series with one and two time constants (two and four DOFs, respectively) were also fitted to the experimental data. We thus compared results for four Prony series.

\section{Elastic part}

As explained in Sec. II C, there are two possibilities for characterizing the elastic part in Eq. (1): the instantaneous response or the long-term response [Eqs. (2) and (3), respectively]. In these two limiting conditions, the viscous nature of the material does not contribute to the material response. However, because of practical limitations neither the instantaneous response nor the long-term response of a material is directly measurable (e.g., Wu et al., 2003).

According to the test protocol of Cheng et al. (2007), the loading time was approximately $10 \mathrm{~s}$ for the stressstretch tests. Compared with the relaxation data, in which about $10 \%$ of the stress is relaxed within $1 \mathrm{~s}$, the 10 -s loading time is not fast enough to be considered as an instantaneous response. It is also not slow enough to be considered as a long-term response because more than $100 \mathrm{~s}$ is needed to reach a plateau on the relaxation curve. Therefore, in the reported standard loading results the viscous behavior of the material contributed to the shapes of the stress-stretch curves. If we obtain the elastic parameters by fitting the Ogden model to the loading data and then use these parameters as the instantaneous response for the elastic part of the non-linear viscoelastic model, the resultant stress-stretch curve will be lower than the experimental data. On the other hand, if the elastic parameters are used as the long-term response then the resultant stress-stretch curve will be higher than the experimental data. In either case, therefore, we must adjust the Ogden parameters to reproduce the experimental curves. We have chosen to take the elastic part of the model to be the instantaneous elastic response and the Prony series of Eq. (2) is therefore used as the time-dependent part.

As usual in such cases, an iterative technique is used here to determine the elastic parameters of the formulation. We use a locally developed program that minimizes an externally computed function using the algorithm of Hooke and Jeeves (1961). In this procedure, the initial values of the parameters $\alpha$ and $\mu$ are those obtained by curve-fitting without taking viscoelasticity into account, as described above. The function to be minimized is implemented as a MATLAB script that invokes FEBio to run a NLV simulation for a given $\alpha$ and $\mu$ and then computes the root-mean- square error (RMSE) between the experimental data and the simulation results,

$$
\operatorname{RMSE}=\sqrt{\left(\frac{\sum_{i=1}^{N}\left(e_{i}-m_{i}\right)^{2}}{N}\right)},
$$

where $e_{i}$ and $m_{i}$ are the experimental and modeling results, respectively, at points where mean values and standard deviations were reported, and $N$ is the number of points. A starting values for the step size $\Delta$ and a minimum step size $\delta$ must be defined. The subroutine is then called repeatedly until $\Delta<\delta$. Since the accuracy of the initial parameters was on the order of $0.01, \Delta$ and $\delta$ were set to 0.01 and 0.001 , respectively. For each function evaluation within the iterative minimization algorithm, the program invokes the MATLAB script and reads the resulting RMSE value. The iteration continues until the stopping criterion is met. The parameters obtained will be referred to below as adjusted Ogden parameters.

To determine what bulk modulus is required to enforce the assumption of incompressibility, we performed a sensitivity analysis to test how our model is affected by variations in the bulk modulus. We observed that for values of the bulk modulus greater than about $10^{3} \mathrm{MPa}($ i.e., $K / \mu \approx 2500$ ) the output stress is constant to within less than $0.4 \%$, meaning that the TM is approximately incompressible.

\section{RESULTS}

\section{A. Parameter identification}

As described in Sec. II D 1, Prony series for the timedependent part of the model were computed for one, two, and three terms, with the three-term series having either six or three DOFs. These four sets of parameters are listed in Table I. For each term of a Prony series, one time constant $\left(\tau_{i}\right)$ and one relaxation coefficient $\left(g_{i}\right)$ are required. For the six-DOF three-term series the $\tau_{i}$ are fairly close to the values of 1,10 , and $100 \mathrm{~s}$ assigned for the three-DOF three-term series, and the corresponding values of $g_{i}$ are very similar in the two cases. This supports the rationale for predefining the time constants. The RMSEs are included as an indication of how much the fitted curves deviate from the experimental data points.

TABLE I. Calculated Prony-series parameters $g_{i}$ and $\tau_{i}$ for relaxation data. RMSE is the root-mean-square error between the fitted curves and the experimental data.

\begin{tabular}{lcccc}
\hline \hline Parameters & 1 term & 2 terms & 3 terms & $\begin{array}{c}3 \text { terms with } \\
\text { predefined time constants }\end{array}$ \\
\hline$g_{1}$ & 0.35 & 0.22 & 0.18 & 0.19 \\
$\tau_{1}(\mathrm{~s})$ & 6.39 & 1.23 & 0.98 & $1^{\mathrm{a}}$ \\
$g_{2}$ & - & 0.13 & 0.08 & 0.07 \\
$\tau_{2}(\mathrm{~s})$ & - & 42.4 & 6.6 & $10^{\mathrm{a}}$ \\
$g_{3}$ & - & - & 0.12 & 0.11 \\
$\tau_{3}(\mathrm{~s})$ & - & - & 91.7 & $100^{\mathrm{a}}$ \\
RMSE & $37.0 \times 10^{-3}$ & $4.0 \times 10^{-3}$ & $0.9 \times 10^{-3}$ & $1.7 \times 10^{-3}$ \\
\hline \hline
\end{tabular}

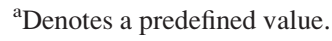


TABLE II. Calculated parameters $\mu$ and $\alpha$ for the Ogden models for three individual TMs (TM\#1-3) and the mean value of the stress-stretch relationship for eleven TM specimens from experimentally reported stress-stretch curves [Cheng et al., 2007, Figs. 4(a)-4(c) and 6(b)].

\begin{tabular}{|c|c|c|c|c|c|c|}
\hline & \multicolumn{3}{|c|}{ Initial parameters } & \multicolumn{3}{|c|}{ Adjusted parameters } \\
\hline & $\mu(\mathrm{MPa})$ & $\alpha$ & RMSE (MPa) & $\mu(\mathrm{MPa})$ & $\alpha$ & RMSE (MPa) \\
\hline TM\#1 [Fig. 4(a)] & 0.69 & 22.6 & 0.015 & 0.75 & 22.5 & 0.016 \\
\hline TM\#2 [Fig. 4(b)] & 0.30 & 29.6 & 0.012 & 0.33 & 29.3 & 0.011 \\
\hline TM\#3 [Fig. 4(c)] & 0.36 & 24.0 & 0.010 & 0.39 & 23.8 & 0.010 \\
\hline Mean [Fig. 6(b)] & 0.38 & 28.6 & 0.029 & 0.40 & 28.8 & 0.025 \\
\hline
\end{tabular}

The initial and adjusted Ogden parameters obtained for the loading curves of each of three individual specimens and for one mean curve for eleven specimens [Cheng et al., 2007, Figs. 4(a)-4(c) and 6(b)] are listed in Table II. In this table, the initial parameters are those obtained directly from a curve-fitting procedure without taking viscoelasticity into account, and the adjusted parameters are those obtained by the procedure explained in Sec. II D2. The RMSE values indicate that we are able to account for the viscous effects without reducing the quality of the fit.

\section{B. Simulated uniaxial tests}

In Fig. 2, the mean values for the experimentally measured stress-stretch data of Cheng et al. [2007, Fig. 6(b)] are shown together with the simulation results for the NLV model with both initial elastic parameters and adjusted elastic parameters. The stress values for the NLV model but with the initial elastic parameters are lower than the experimental data, while the NLV model with the adjusted elastic parameters matches the data well. An elastic response (with the adjusted elastic parameters but no relaxation) is also shown in Fig. 2; this response is, as expected, higher than the experimental data.

Figure 3 shows the experimentally reported relaxation data of Cheng et al. (2007, Fig. 8) together with the simulated relaxation results of the NLV model with adjusted parameters, for one-term, two-term, and three-term Prony

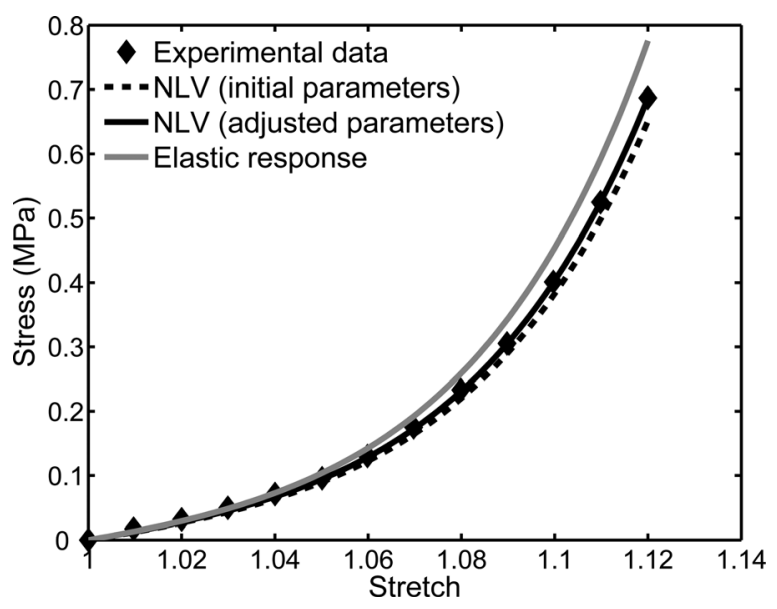

FIG. 2. Stress-stretch relationship for the mean experimental data of Cheng et al. (2007, Fig. 6) and for the NLV model with initial parameter values, with adjusted parameters, and with adjusted parameters but no relaxation (i.e., elastic response).

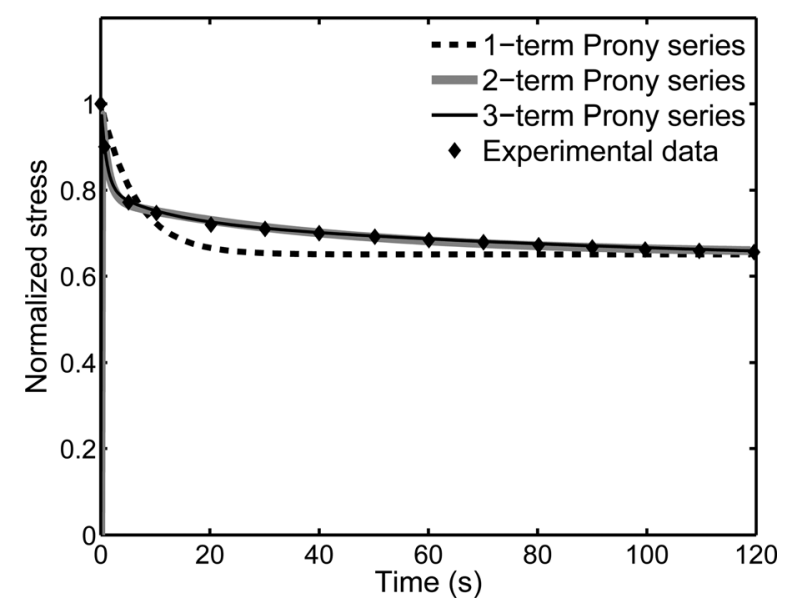

FIG. 3. Experimental data (Cheng et al., 2007, Fig. 8) and comparison with simulated relaxation tests with one-term, two-term, and three-term Prony series.

series (after normalizing by the maximum stress). [Since, for the three-term series, the three-DOF version (with predefined time constants) and the six-DOF version produce very similar responses, for the sake of clarity only the three-DOF version is included in this figure.] It can be seen that the normalized stress for the one-term Prony series does not provide a good representation of the experimental data (with an RMSE of $3.7 \%$ of the normalized maximum stress) while the two-term and three-term Prony series match the experimental data quite well (with RMSEs of 0.4 and $0.17 \%$ of normalized maximum stress, respectively).

\section{Loading and unloading curves}

One of the important criteria in evaluating a viscoelastic model is its ability to predict both the loading behavior and the unloading behavior with the same material parameters. Cheng et al. (2007, Fig. 4) reported loading and unloading curves for three individual TM specimens. Three sets of Ogden parameters were obtained based only on the loading curves of three individual TM specimens as explained in Sec. IID 2, for the elastic part of the model. Since no relaxation data were reported for individual specimens, the parameters of the three-term Prony series, with predefined time constants, were obtained for average relaxation data for the viscous part of the model. The NLV model was then used to simulate the unloading behavior. In Fig. 4 the three sets of experimental loading and unloading curves are shown together with the simulation results for the three corresponding NLV models. The simulation results match the experimental data quite well, the RMSEs of the combined loading and unloading curves being $1.5 \%, 2.9 \%$, and $2.4 \%$ of the maximum stress magnitudes for TM's 1, 2, and 3, respectively. The most noticeable discrepancy is that the simulated unloading curves pass below the zero stress level; this is discussed in Sec. IV.

Note that, although the elastic parameters of the three TMs were obtained from the individual loading curves of the three reported samples, the viscous parameters were obtained from the mean relaxation curves for nine TMs, because individual relaxation curves were not available. In addition, the simulated loading and unloading times were 

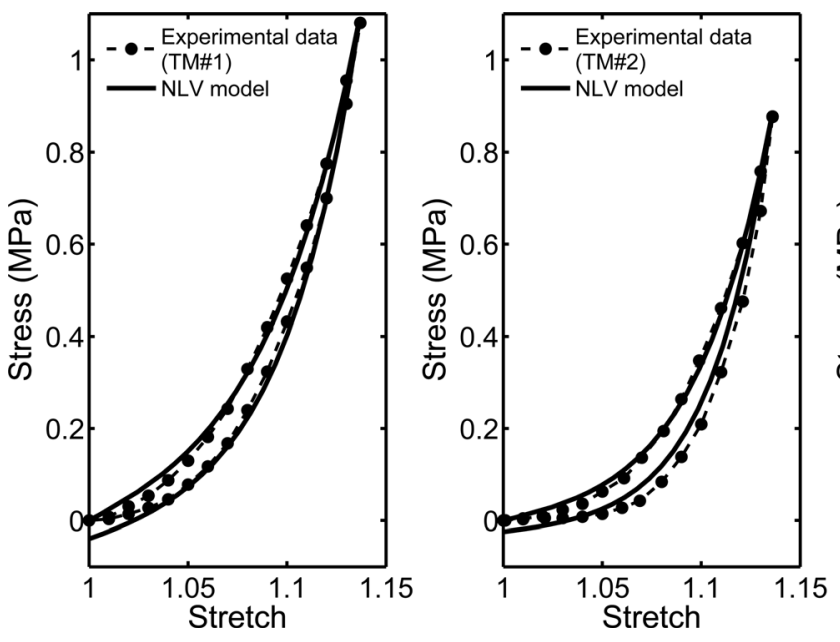

calculated based on the average dimensions of the eleven specimens of Cheng et al. (2007) because the dimensions were not reported for individual specimens. This use of mean data to determine the parameters of individual models may account for some of the discrepancies seen in Fig. 4.

\section{Strain-rate effect}

In the experimental relaxation curve (Fig. 3), the normalized stress decreases by almost $10 \%$ in less than $1 \mathrm{~s}$ after the peak. Since according to the experimental protocol described by Cheng et al. (2007) the loading time was $\sim 0.54 \mathrm{~s}$, the results might have been significantly affected by the relaxation that occurred during the loading time. We have investigated the sensitivity of the NLV model to strain rate by varying the loading time (the time required to apply the specified deformation in the relaxation test) in simulations. As shown in Figs. 5(a) and 5(b) the long-term response of the model is not affected by changing the loading time. The short-term response, however, is affected as shown in Fig. 5(a) and more clearly in Fig. 5(b), which focuses on the first $12 \mathrm{~s}$, where the peak stress changes by less than $2.5 \%$ for loading times between 0.01 and $0.54 \mathrm{~s}$.

\section{DISCUSSION}

\section{A. Non-linear viscoelastic model in the frequency domain}

It is often instructive to explore the implications of a viscoelastic model in the frequency domain. For example,

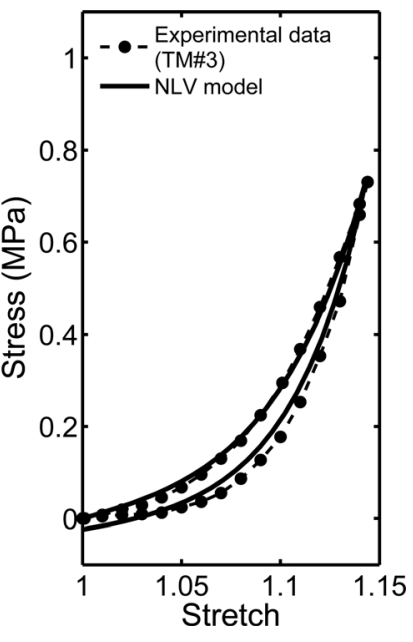

FIG. 4. Experimental data for three individual TM specimens reported by Cheng et al. [2007, Figs. 4(a)-4(c)] compared with simulated results from NLV model.

Fung (1993) showed, for a linear viscoelastic model with one time constant, that at low frequencies the Young's modulus remains constant at its static value, but at a certain critical frequency the modulus rises sharply before settling at a new high-frequency value. For a non-linear viscoelastic model, Charlebois et al. (2013) reported that the stored energy shows a similar stiffening pattern over the frequency domain.

To demonstrate how stress-stretch relationships and hysteresis loops are affected by the loading frequency, a harmonic displacement-controlled loading condition was modeled at three circular frequencies, 0.001, 0.1, and $10 \mathrm{rad} / \mathrm{s}$, for a three-term Prony series. The results are shown in Fig. 6. Note that the maximum stress magnitude for the frequency of $\omega=10 \mathrm{rad} / \mathrm{s}$ is higher than that for the frequencies of $\omega=0.1$ and $0.001 \mathrm{rad} / \mathrm{s}$, which demonstrates that this viscoelastic model behaves more stiffly at high frequencies than at low frequencies. It can be shown that the maximum stress magnitude approaches plateaus at the lower and upper frequencies (i.e., $\sim 0.001$ and $10 \mathrm{rad} / \mathrm{s}$, respectively), as described in the previous paragraph. In addition, it can be seen that the area inside the hysteresis loop (i.e., the area between the loading and unloading curves, representing lost energy) is larger at the middle frequency (i.e., $\omega=0.1 \mathrm{rad} / \mathrm{s}$ ) than at the upper and lower frequencies (i.e., $\omega=10$ and $0.001 \mathrm{rad} / \mathrm{s}$, respectively). (The lost energy spectra in the frequency domain will be discussed below in more detail.)

At each frequency, a steady state is obtained after a few cycles of loading and unloading. The number of cycles

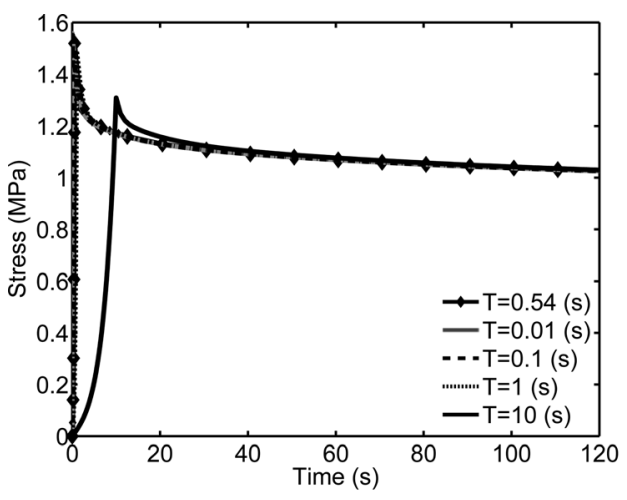

a

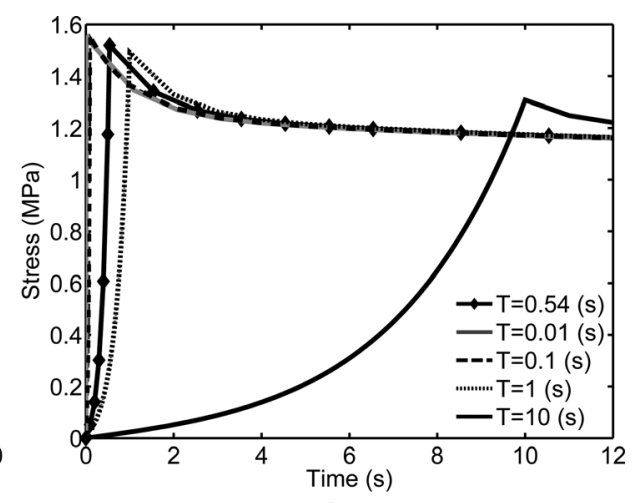

b
FIG. 5. Effect of loading time on simulated relaxation response of TM strips. (a) Full duration of measurements (120 s), showing lack of long-term differences. (b) Expanded view of first $12 \mathrm{~s}$ of response, emphasizing the short-term differences. 


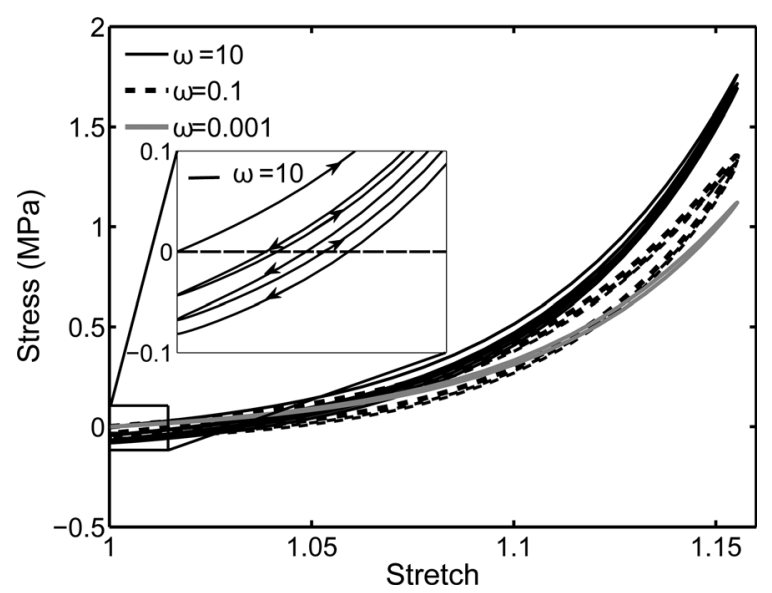

FIG. 6. Frequency effects on stress-stretch relationship. The first three cycles of hysteresis loop are shown for each of three circular frequencies. The inset shows a magnified view of the area around the origin of the stretch and stress axes for $\omega=10 \mathrm{rad} / \mathrm{s}$, to demonstrate the changes in the loading and unloading paths from one cycle to the next.

required to reach steady state is smaller at low frequencies than at high frequencies (cf. Schatzmann et al., 1998; Charlebois et al., 2013). Figure 6 shows that the steady-state closed loops are reached within the first cycle for the lowest frequency and are almost reached by the third cycle for the middle frequency, but are not yet reached after three cycles for the highest frequency.

As mentioned in Sec. II, it is very common to choose one relaxation time per decade of data. As we increase the number of time constants the spectrum of the lost energy becomes more uniform (e.g., Fung, 1993), which is desirable for describing the usual time-dependent behavior of biological tissues. Thus, although two time constants seem to fit the relaxation data as well as three (Fig. 3), a three-time-constant series is probably a better choice to model the TM. To illustrate this, Fig. 7(a) shows lost-energy spectra calculated from hysteresis loops using the method described by Charlebois et al. (2013). The lost-energy spectra were computed for the viscous parameters shown in Table I (i.e., for one-term, two-term, and three-term Prony series, with predefined time constants for the latter) and the elastic parameters shown in Table II [i.e., the adjusted parameters for the mean elastic response of Cheng et al., 2007, Fig. 6(b)]. As seen in this figure, increasing the number of Prony-series terms results in a more uniform lost-energy spectrum over the frequency range of approximately $0.006-4 \mathrm{rad} / \mathrm{s}$. In addition, Fig. 7 demonstrates that at low frequencies (i.e., $\omega<0.001 \mathrm{rad} / \mathrm{s}$ ) and high frequencies (i.e., $\omega>10 \mathrm{rad} / \mathrm{s}$ ) the lost energy becomes small. This means that the model behaves like an elastic material at frequencies far beyond these values, so it will no longer be sensitive to strain rate. This explains why the relaxation behavior of this model is not sensitive to loading rates higher than $10 \mathrm{~s}^{-1}$ (i.e., loading times less than $0.1 \mathrm{~s}$, as shown in Fig. 5).

For a linear viscoelastic model, a complex dynamic modulus is defined which is composed of storage and loss components (Mase and Mase, 1999). It can be shown that, for a linear viscoelastic model with a Prony series representing the viscous part, the peaks of the loss modulus and of the lost-energy spectrum occur at the same frequencies (e.g., Park and Schapery, 1999). Such a modulus is not defined for a non-linear viscoelastic model, but the lost-energy spectrum peaks for the non-linear viscoelastic model occur at the same frequencies as are predicted by a linear viscoelastic model with the same viscous parameters. To demonstrate the contribution of each term of the Prony series to the total lostenergy spectrum, the model with a three-term Prony series (with predefined time constants) is analyzed separately in Fig. 7(b). As this figure shows, each term has a peak occurring at an angular frequency corresponding to the inverse of its time constant (i.e., $\omega_{1}=1 / \tau_{1}=1, \omega_{2}=1 / \tau_{2}=10$, and $\left.\omega_{3}=1 / \tau_{3}=100\right)$. Note that the height of each peak depends on the value of the associated $g_{i}$.

\section{B. Loading and unloading loops}

As a result of viscoelastic processes, at the end of each unloading process the length of the TM strip would tend to be longer than its initial length. This means that to force the specimen back to its initial length we would need to apply a compressive stress. In the experimental tests, the TM strips do not resist the compression and some local buckling happens, so the loading and unloading curves form a closed loop. However, with the numerical model no buckling occurs and, as Fig. 4 shows, for a single cycle the loading and unloading curves do not form a closed loop. Loading and unloading loops approach a closed configuration over a number cycles, as discussed above in connection with Fig. 6.

\section{Conclusion}

The material parameters (Tables I and II) for a nonlinear viscoelastic model of human TM were derived from

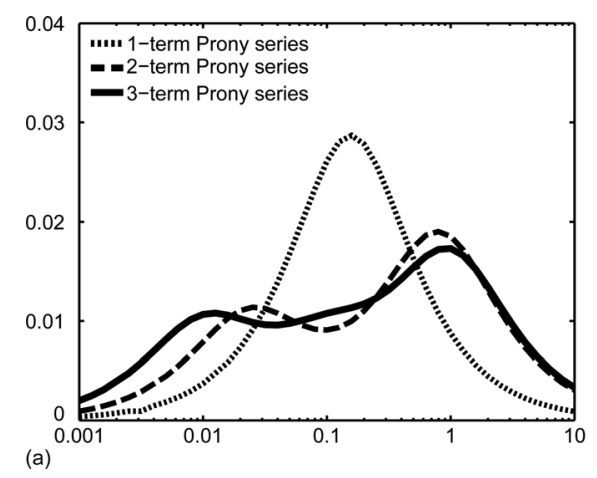

J. Acoust. Soc. Am., Vol. 134, No. 6, December 2013

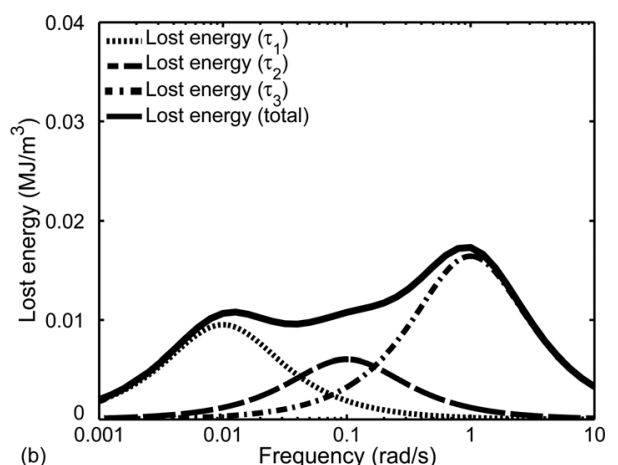

Motallebzadeh et al:: Tympanic-membrane non-linear viscoelasticity 
experimental data reported by Cheng et al. (2007) and the model is able to reproduce the results of their mechanical tests. Most significantly, this allows us for the first time to model both a loading curve and the corresponding unloading curve with a single set of parameters.

This model is suitable for large deformations of the TM and for frequencies in the range of about $0.003-3 \mathrm{rad} / \mathrm{s}$ (approximately $0.001-0.64 \mathrm{~Hz}$ ). These conditions correspond to those involved in tympanometry, in which a large sweeping ear-canal pressure (e.g., -300 to $+200 \mathrm{daPa}$ ) is applied in less than $10 \mathrm{~s}$.

\section{ACKNOWLEDGMENTS}

This work was supported by the Canadian Institutes of Health Research and the Natural Sciences and Engineering Research Council of Canada.

Aernouts, J., and Dirckx, J. J. J. (2012). "Static versus dynamic gerbil tympanic membrane elasticity: Derivation of the complex modulus," Biomech. Model. Mechanobiol. 11, 829-840.

Békésy, G. v. (1949). "The structure of the middle ear and the hearing of one's own voice by bone conduction," J. Acoust. Soc. Am. 21, 217-232.

Charlebois, M., Motallebzadeh, H., and Funnell, W. R. J. (2013). "Viscohyperelastic law for finite deformations: a frequency analysis," Biomech. Model. Mechanobiol. 12, 705-715.

Cheng, T., Dai, C., and Gan, R. Z. (2007). "Viscoelastic properties of human tympanic membrane," Ann. Biomed. Eng. 35, 305-314.

Daphalapurkar, N. P., Dai, C., Gan, R. Z., and Lu, H. (2009). "Characterization of the linearly viscoelastic behavior of human tympanic membrane by nanoindentation," J. Mech. Behav. Biomed. Mater. 2, $82-92$.

Decraemer, W., Maes, M., and Vanhuyse, V. (1980a). "An elastic stressstrain relation for soft biological tissues based on a structural model," J. Biomech. 13, 463-468.

Decraemer, W. F., Maes, M. A., Vanhuyse, V. J., and Vanpeperstraete, P. (1980b). "A non-linear viscoelastic constitutive equation for soft biological tissues, based upon a structural model," J. Biomech. 13, 559-564.

Fay, J., Puria, S., Decraemer, W. F., and Steele, C. (2005). "Three approaches for estimating the elastic modulus of the tympanic membrane," J. Biomech. 38, 1807-1815.

Feldman, A. S. (1974). "Eardrum abnormality and the measurement of middle ear function," Arch. Otolaryngol. Neck Surg. 99, 211-217.

Fung, Y. (1993). Biomechanics: Mechanical Properties of Living Tissues, 2nd ed. (Springer, New York), pp. 482-483.

Hooke, R., and Jeeves, T. A. (1961). "“Direct Search' Solution of numerical and statistical problems," J. ACM 8, 212-229.

Huang, G., Daphalapurkar, N. P., Gan, R. Z., and Lu, H. (2008). "A method for measuring linearly viscoelastic properties of human tympanic membrane using nanoindentation,” J. Biomech. Eng. 130, 014501.

Humphrey, J. (2003). "Continuum biomechanics of soft biological tissues," Proc. R. Soc. London, Ser. A 459, 3-46.
Khanna, S. M., and Tonndorf, J. (1972). "Tympanic membrane vibrations in cats studied by time-averaged holography," J. Acoust. Soc. Am. 51, 1904-1920.

Kirikae, I. (1960). The Structure and Function of the Middle Ear (University of Tokyo Press, Tokyo), pp. 46-48.

Knauss, W. G., and Zhao, J. (2007). "Improved relaxation time coverage in ramp-strain histories," Mech. Time-Depend. Mater. 11, 199-216.

Kuypers, L. C., Decraemer, W. F., and Dirckx, J. J. J. (2006). "Thickness distribution of fresh and preserved human eardrums measured with confocal microscopy," Otol. \& Neurotol. 27, 256-264.

Ladak, H. M., Funnell, W. R. J., Decraemer, W. F., and Dirckx, J. J. (2006). "A geometrically nonlinear finite-element model of the cat eardrum," J. Acoust. Soc. Am. 119, 2859-2868.

Lim, D. J. (1995). "Structure and function of the tympanic membrane: A review," Acta Otorhinolaryngol. Belg. 49, 101-115.

Luo, H., Dai, C., Gan, R. Z., and Lu, H. (2009a). "Measurement of young's modulus of human tympanic membrane at high strain rates," J. Biomech. Eng. 131, 064501.

Luo, H., Lu, H., Dai, C., and Gan, R. Z. (2009b). "A comparison of Young's modulus for normal and diseased human eardrums at high strain rates," Int. J. Exp. Comput. Biomech. 1, 1-22.

Maas, S. A., Ellis, B. J., Ateshian, G. A., and Weiss, J. A. (2012). "FEBio: finite elements for biomechanics," J. Biomech. Eng. 134, 011005.

Martins, P., Natal Jorge, R., and Ferreira, A. (2006). "A comparative study of several material models for prediction of hyperelastic properties: Application to silicone-rubber and soft tissues," Strain 42, $135-147$.

Mase, G. T., and Mase, G. E. (1999). Continuum Mechanics for Engineers, 2nd ed. (CRC, New York), pp. 344-346.

Ogden, R. W. (1972). "Large deformation isotropic elasticity-on the correlation of theory and experiment for incompressible rubberlike solids," Proc. R. Soc. London, Ser. A 326, 565-584.

Park, S. W., and Schapery, R. A. (1999). "Methods of interconversion between linear viscoelastic material functions, Part I-A numerical method based on Prony series," Int. J. Solids Struct. 36, 1653-1675.

Puso, M. A., and Weiss, J. A. (1998). "Finite element implementation of anisotropic quasi-linear viscoelasticity using a discrete spectrum approximation," J. Biomech. Eng. 120, 62-70.

Qi, L., Funnell, W. R. J., and Daniel, S. J. (2008). "A nonlinear finiteelement model of the newborn middle ear," J. Acoust. Soc. Am. 124, 337-347.

Schatzmann, L., Brunner, P., and Stäubli, H. (1998). "Effect of cyclic preconditioning on the tensile properties of human quadriceps tendons and patellar ligaments," Knee Surg. Sports Traumatol. Arthrosc. 6, 56-61.

Schomburg, W. K. (2011). Introduction to Microsystem Design (Springer, Berlin), pp. 29-30.

Taylor, R. L., Pister, K. S., and Goudreau, G. L. (1970). "Thermomechanical analysis of viscoelastic solids," Int. J. Numer. Methods Eng. 2, 45-59.

Wu, J. Z., Dong, R. G., Smutz, W. P., and Schopper, A. W. (2003). "Nonlinear and viscoelastic characteristics of skin under compression: Experiment and analysis," Biomed. Mater. Eng. 13, 373-385.

Zhang, X., and Gan, R. Z. (2010). "Dynamic properties of human tympanic membrane-experimental measurement and modelling analysis," Int. J. Exp. Comput. Biomech. 1, 252-270. 